\title{
On Deep History and Pyschotropy
}

\author{
Benjamin Campbell
}

Published online: 13 April 2014

(C) Springer Science+Business Media New York 2014

Four field anthropology, often considered as a lesser form of anthropology done in backwater departments, is in fact a serious endeavor in its own right. It requires one to let down the narrow boundaries of one's sub-disciplinary assumptions and see the forest of the human species through the trees of different epoch, areas, geographies, cultures, social structures, and political regimes. It takes certain mixture of naivety, and ambition to attempt such an undertaking.

Or more likely, someone outside the field. If you want to see what such a four field anthropology might look like, you can find it in Daniel Smail's Deep History and the Brain. Smail, dissatisfied with the traditional perspective of the historian, which he finds increasingly narrow and time bound, attempts to find a principle by which he can survey all of human history, from the origin of the species to the origin of civilization to the origins of our own modern age.

What factor, you ask, could possibly unite the cultural change apparent over such depths of time, not to mention the cultural diversity evident in different parts of the world? If you have been reading our species' self-generating press clippings, it is our large brain. The brain that is the seat of the intelligence that has created the technology that changes our physical environment and generates the social structures that accommodate us to those new environments. In other words, our brain is the one thing that remains the one apparently identifiable biological factor common to all of the tremendous variety of ways in which we human live.

Not surprising then that Smail would look to the brain to find a new explanatory principle by which to write history. And in the process stumbles upon what has been considered the purview of four field anthropology. Smail contrasts his focus on the brain with the old ways of writing history, including a focus on documents and the belief that human history did not begin until civilization. The former point may be novel for historians in practicing their craft, (though surely some have accepted oral

B. Campbell (两)

Department of Anthropology, University of Wisconsin, Milwaukee, USA

e-mail: campbelb@uwm.edu 
history as a meaningful, if marginalized exercise). It is the latter point, the depth of our humanity, that should most directly engage anthropologists to consider the value of a four field anthropology.

We anthropologists have produced the ethnographic and archeological evidence that demonstrates the humanity common to hunter-gather, horticultural and pastoral societies, all of which existed prior to civilization. Yet, I find myself having to remind my students and the general public again and again, about both the width and depth of our humanity. Smail's complaint about the increasingly narrow time depth may or may not be true of historians, but it does characterize our society as a whole. As a culture, we are so identified with our own western industrialized educated democratic existence that we tend to think of our own experience as defining humanity, in much the same way that many traditional societies refer to themselves as the people, meaning the human ones.

Hence, the larger mission of anthropology, to present the ways in which a single human species can experience their essential humanity in different cultural forms. So even if a book like On Deep History and the Brain is in some ways naïve and superficial, it should be thought-provoking to anthropologists interested in how we can create a deeper and rich version of what Smail points toward.

I see two issues of particular interest. The first is the substantive issue of how a single species can live in so many different cultural environments and yet still share a single biological identity. This question is currently being taken up by the emerging field of Neuroanthropology. Neuroanthropology seeks to capitalize on the recent explosion in neuroscientific knowledge to understand how cultural conditions both shape and are shaped by a neuroplastic brain (see Lende and Downey 2012). In other words, a brain centered understanding of the interactive nature of human biology and culture.

For a specific example, the 7 repeat allele (7R) of the DRD4 dopamine receptor reduces the sensitivity of dopamine to dopaminergic transmission, resulting in a weaker dopamine signal in the prefrontal cortex. By itself the DRD4 7R+ allele has been related to an increased risk of ADHD (Wu et al. 2012), a socially problematic condition. But more recently, it has become clear from studies in U.S. and Europe that DRD4 is best understood not as a risk-conferring gene but a plasticity gene (Belsky et al. 2009). The presence of the DR4 $7 \mathrm{R}+$ allele leads to a differential impact of experience in early childhood on later life. In one example, in a sample of Australian adults in their early 30's those individuals without a DRD4 7R+ allele, an increasing number of childhood adversities lead to reduced resilience in adulthood, as might be expected. In contrast, among those individuals with a $7 \mathrm{R}+$ allele there was no relationship between childhood adversity and adult resilience (Das et al. 2011).

There are similar findings regarding the relationship of other brain plasticity genes such as the serotonin transporter 5HTTLPR (which removes serotonin from the synapse) and BDNF (brain derived neurotrophic factor-which does what the name implies) with depression (Aguilera et al. 2009; Carver et al. 2011). Another gene that may play a similar role in later behavior (in this case antisocial behavior) in response to the childhood environment is that for mono amine oxidase (MAO-A) (Fergusson et al. 2012). The implication is clear, individual variation within a 
cultural is inherently dynamic and biocultural because it represents the interaction of variable cultural environments and a plastic brain, as marked by certain genes.

The question for anthropologists is to understand how variation between cultures interacts with brain plasticity to generate reliable differences between cultures. Childhood adversity may be a very good place to start such an investigation, because human brains clearly expect to develop in the context of consistent and supportive parenting. Given the ubiquity of childhood adversity cross-culturally, its hard not to imagine that differences in the frequency of any number of neuroplastic genes across populations will interact with childhood adversity to generate cross cultural variation in individual well-being.

Aside from raising the question of how the human brain interacts with experience to generate human cultural variation, Smail's book also represents a counter current against the grain of history, which he suggests is as much a cultural blinder as it is a deeper understanding of ourselves and our past. In other words the book is a sort of cultural critique (in the sense of a perspective from outside our own culture) of the way we understand ourselves as humans. And cultural critique is always close to the heart of anthropology and anthropologists.

With that thought firmly in mind, the particular part of Smail's focus on the brain and history that has captured the attention of Michael Oldani and Stefan Ecks in putting together the papers in this volume is "psychotropy." Pyschotropy refers to the way in which different cultures manipulate the basic mechanisms of the brain toward achieving satisfaction, whether that manipulation is through changing the environment, enhancing everyday experiences, such as food, sex, and social behavior, or by ingesting specific chemical substances. Regardless of where they start each of these behaviors ends up having its final effects through how it impacts the brain's operation.

Smail distinguishes two basic types of psychotropy. The first is telotropy in which individual brain states are influenced by those outside of themselves (social dominance would be a good example). The other is autotropy in which an individual influences their own brain states (ingesting food or chemical substances are the obvious examples). These are not particularly new ideas. Primatologists have a reasonably detailed understanding of how dominant individuals in primate groups influence the levels of neurotransmitters in subordinate members of the group (see Michopoulos et al. 2009) for an interesting example) and hence alter their behavioral tendencies. Nor is it difficult to draw parallels with the impact of public bodily dismemberment of one sort or another for traitors in medieval Europe for example. In a more autotropic vein, The Annale school noticed that either tea and coffee tended to be the drink of choice in different cultures, a fact explained by the need for only one twinkle on the tongue.

But social dominance is an unavoidable fact of life and a twinkling on the tongue is a relatively minor reward unlikely to re-establish individual or cultural priorities. What happens when you have substances that can set your whole brain a buzzing, like the psychoactive pharmaceuticals in our society? Yes, the idea of psychotropy gains far more traction. The drugs are powerful enough to represent a novel element that can decouple the feeling of reward from the actions with which they are usually associated. As such they appear capable of a generating an entirely new set of 
dynamic associations, all within the existing cultural paradigm. Addictions to narcotics, self-medication with alcohol, the use of Ritalin to increase concentration while studying, anti-depressants, and so forth, are all obvious examples of how substances may have an autotrophic impact on neurotransmitters, eclipsing the telotrophic influences found in everyday life.

Of course, anthropologists recognize that the use of powerful psychoactive substances, including those that are culturally sanctioned, is not confined to our modern society by any means. Throughout history, cultures have used a variety of powerful pyschoactive drugs, including ayahuasca, peyote, psilocybin mushroom, amanita muscaria and cannabis, among others. Most often it is in the support of healing or religious rituals. These rituals represent a means of what Tiger and McGuire (2010) refer to as "brain soothing," not just for the individuals but for the collected group.

So at the same time that we consider the brain mechanisms that make psychotropy possible, we have to ask how it is that the use of pharmaceutical drugs, substances of addiction and consumerism reflect an overarching cultural zeitgeist that makes such behaviors common, AND influences our perception of their meaning. For instance, given the great effort put into advertising psychoactive pharmaceuticals in today's society, the drugs' ubiquity surely reflects larger cultural, economic and political forces as much as their efficacy in reducing undesirable somatic sensations and/or ideations. It is those larger waves of culture that shape our perception of the drugs as a desirable mechanism of psychotropy. Just to drive the point home, advertisements extolling the benefits of adding a second antidepressant on top of one that is not providing adequate relief clearly demonstrate the cultural logic of pharmaceutical psychotropy. It would be equally logical to suggest discontinuing the use of the first anti-depressant if it is not providing adequate relief, but that is not consistent with our cultural belief that a pill can make you happy.

It is a common argument among anthropologists that the same globalizing economic and political processes that generate large amounts of psychoactive substances and distributed them widely are also associated with the production of greater social inequality. Furthermore its axiomatic among biocultural anthropologists that the process of westernization disrupts the more equalitarian social networks that characterize humans in subsistence societies as hunter gathers, creating social isolation and increased distress and anxiety. It is the increased anxiety and depression that seems to be particularly crippling in our society and most likely to elicit the logical of modern psychotropy. Leaving out the pharmaceutical psychoactive drugs for the moment, look at the recent rise of legalized marijuana in Colorado, California, Washington and Uruguay. THC, the active ingredient in marijuana, is now known to act through the endocannabinoid receptors as part of a system involved with anxiety (Viveros et al. 2005). As part of its psychoactive properties, marijuana can reduce anxiety. So even the counter culture's choice of psychotropy appears to be related to the larger cultural zeitgeist. Now that is hegemonic!

Ideas that aim to provide an overarching rationale for variety - in this case cultural variety-based on the creative combination of just a few factors can be very 
satisfying — it might even be called psychotropic — to some minds. But of course, the proof of a concept like psychotropy is not in the sweep we impart to human history, but in the presentation of specific practices and beliefs, and behaviors in specific cultural contexts. This volume presents a variety of initial cases that attempt to do just that, ranging from the pharmacological treatment of addicts in recovery to prescription patterns for antidepressants in the Upper Peninsula of Michigan to the way in which Indian psychiatry frames women's mental health issues. The reader will have to decide for themselves how well the notion of psychotropy both illuminates the cases and points out their essential similarities.

\section{References}

Aguilera, M., B. Arias, M. Wichers, N. Barrantes-Vidal, J. Moya, H. Villa, J. van Os, M.I. Ibáñez, M.A. Ruipérez, G. Ortet, and L. Fañanás

2009 Early Adversity and 5-HTT/BDNF Genes: New Evidence of Gene-Environment Interactions on Depressive Symptoms in a General Population. Psychological Medicine 39: 1425-1432.

Belsky, J., C. Jonassaint, M. Pluess, M. Stanton, B. Brummett, and R. Williams

2009 Vulnerability Genes or Plasticity Genes? Molecular Psychiatry 2009(14): 746-754.

Carver, C.S., S.L. Johnson, J. Joormann, J. Lemoult, and M.L. Cuccaro

2011 Childhood Adversity Interacts Separately with 5-HTTLPR and BDNF to Predict Lifetime Depression Diagnosis. Journal of Affective Disorders 132: 89-93.

Das, D., N. Cherbuin, X. Tan, K.J. Anstey, and S. Easteal

2011 DRD4-exonIII-VNTR Moderates the Effect of Childhood Adversities on Emotional Resilience in Young-Adults. PLoS One 6: e20177.

Fergusson, D.M., J.M. Boden, L.J. Horwood, A. Miller, and M.A. Kennedy

2012 Moderating Role of the MAOA Genotype in Antisocial Behaviour. The British Journal of Psychiatry 200: 116-123.

Lende, D., and G. Downey (eds)

2012 The Encultured Brain: An Introduction to Neuroanthropology. MIT Press.

Michopoulos, V., S.L. Berga, J.R. Kaplan, and M.E. Wilson

2009 Social Subordination and Polymorphisms in the Gene Encoding the Serotonin Transporter Enhance Estradiol Inhibition of Luteinizing Hormone Secretion in Female Rhesus Monkeys. Biology of Reproduction 81: 1154-1163.

Tiger, L., and M. McGuire

2010 God's Brain. Prometheus Books.

Viveros, M.P., E.M. Marco, and S.E. File

2005 Endocannabinoid System and Stress and Anxiety Responses. Pharmacology Biochemistry Behavior 81: 331-342.

Wu, J., H. Xiao, H. Sun, L. Zou, and L.Q. Zhu

2012 Role of Dopamine Receptors in ADHD: A Systematic Meta-Analysis. Molecular Neurobiology 45: 605-620. 